\title{
Segmentation of photospheric magnetic elements corresponding to coronal features to understand the EUV and UV irradiance variability ${ }^{\star}$
}

\author{
J. J. Zender ${ }^{1}$, R. Kariyappa ${ }^{2}$, G. Giono ${ }^{3}$, M. Bergmann ${ }^{4}$, V. Delouille ${ }^{5}$, L. Damé ${ }^{6}$, J.-F. Hochedez ${ }^{6,5}$, and S. T. Kumara ${ }^{7}$
}

${ }^{1}$ European Space Research and Technology Center (ESTEC), 2200 AG Noordwijk, The Netherlands e-mail: Joe.Zender@esa.int

2 Department of Physics and R\&D Division, Vemana Institute of Technology, 560034 Bangalore, India

3 Department of Space and Plasma Physics, School of Electrical Engineering, Royal Institute of Technology KTH, 10044 Stockholm, Sweden

4 Julius-Maximilians-Universität Würzburg, Institut für Informatik, 97074 Würzburg, Germany

5 Royal Observatory of Belgium, Circular Avenue 3, 1180 Brussels, Belgium

6 LATMOS (Laboratoire Atmosphères, Milieux, Observations Spatiales), 11 boulevard d'Alembert, 78280 Guyancourt, France

7 Department of Physics, APS College of Engineering, 560082 Bangalore, India

Received 18 October 2016 / Accepted 27 May 2017

\begin{abstract}
Context. The magnetic field plays a dominant role in the solar irradiance variability. Determining the contribution of various magnetic features to this variability is important in the context of heliospheric studies and Sun-Earth connection.

Aims. We studied the solar irradiance variability and its association with the underlying magnetic field for a period of five years (January 2011-January 2016). We used observations from the Large Yield Radiometer (LYRA), the Sun Watcher with Active Pixel System detector and Image Processing (SWAP) on board PROBA2, the Atmospheric Imaging Assembly (AIA), and the Helioseismic and Magnetic Imager (HMI) on board the Solar Dynamics Observatory (SDO).

Methods. The Spatial Possibilistic Clustering Algorithm (SPoCA) is applied to the extreme ultraviolet (EUV) observations obtained from the AIA to segregate coronal features by creating segmentation maps of active regions (ARs), coronal holes (CHs) and the quiet sun (QS). Further, these maps are applied to the full-disk SWAP intensity images and the full-disk (FD) HMI line-of-sight (LOS) magnetograms to isolate the SWAP coronal features and photospheric magnetic counterparts, respectively. We then computed fulldisk and feature-wise averages of EUV intensity and line of sight (LOS) magnetic flux density over ARs/CHs/QS/FD. The variability in these quantities is compared with that of LYRA irradiance values.

Results. Variations in the quantities resulting from the segmentation, namely the integrated intensity and the total magnetic flux density of ARs/CHs/QS/FD regions, are compared with the LYRA irradiance variations. We find that the EUV intensity over $\mathrm{ARs} / \mathrm{CHs} / \mathrm{QS} / \mathrm{FD}$ is well correlated with the underlying magnetic field. In addition, variations in the full-disk integrated intensity and magnetic flux density values are correlated with the LYRA irradiance variations.

Conclusions. Using the segmented coronal features observed in the EUV wavelengths as proxies to isolate the underlying magnetic structures is demonstrated in this study. Sophisticated feature identification and segmentation tools are important in providing more insights into the role of various magnetic features in both the short- and long-term changes in the solar irradiance.
\end{abstract}

Key words. Sun: magnetic fields - Sun: atmosphere - Sun: corona

\section{Introduction}

Solar magnetic fields are manifested as distinct features on the Sun because their presence cause changes in the radiative properties of the solar atmosphere. The features with high magnetic fields are known as active regions. In the photosphere, active regions may include both dark sunspots with intense magnetic fields and bright faculae with weaker fields, as reviewed in (Solanki 2003). Therefore the average photospheric intensity of an active region depends on its average magnetic flux in a complex way (e.g. Ortiz \& Rast 2005). However, active region intensity at chromospheric and coronal heights generally increases monotonically with increasing magnetic flux. This is presumably

\footnotetext{
$\star$ The movie associated to Fig. 2 is available at http://www . aanda.org
}

because the presence of the magnetic fields causes non-radiative energy to be channeled into the outer atmospheric layers of the Sun (e.g. Klimchuk 2006; Ermolli et al. 2014).

At chromospheric heights the different levels of intensity and magnetic flux lead to a picture of plages, chromospheric network, and bright points as the classes of surface structures associated with measurable magnetic fields on the Sun (e.g. Skumanich et al. 1975). Although variations in the proportions of these various structures on the Sun produce variability in its radiative output, the details of the relationship between magnetic structure evolution and solar spectral irradiance variability is not routinely determined from full-disk magnetograms and intensity images. Even though the magnetic structure evolution and its influence on solar irradiance is not yet carried out on a fully automated basis, first results for the total solar irradiance (TSI) and ultraviolet (UV), visible, and infrared (IR) part 
of the spectrum were made available in recent years (Yeo et al. 2014a,b; Wenzler et al. 2006). Investigations on the UV and the extreme UV (EUV) part of the spectrum has been provided by Haberreiter et al. (2014)

Direct comparison of Ca II $\mathrm{K}$ images and magnetograms shows the strong association between plage and strong field regions (Babcock \& Babcock 1955; Leighton 1959; Howard 1959). Stepanov \& Petrova (1959) gave the first magnetic flux versus $\mathrm{Ca}$ II $\mathrm{K}$ intensity curves measured in calcium faculae (plages) with an electronic magnetograph. Their linear-linear scatter plots are fitted with a smooth, slightly non-linear curve. Skumanich et al. (1975) made similar observations in the quiet Sun and suggested a linear relation between the absolute value of the magnetic flux density and Ca II K-line intensity.

After Ermolli et al. (2009) demonstrated that Ca II K spectroheliogram time series from several observatories can be combined for an intensity distribution analysis of bright features covering timescales longer than one solar cycle, Bertello et al (2016) correlated the Ca II K time series to the sunspot numbers. They found that the two time series match very well with yearly correlation coefficients higher than 0.92. In an overview paper, Ermolli et al. (2014) discuss several solar cycle indices from the photosphere, chromosphere, and several coronal altitudes, and their potential inter-relation.

Pevtsov et al. (2003) have compared the disk-integrated solar X-ray flux to the total unsigned magnetic flux. Using almost $10 \mathrm{yr}$ of soft X-ray data from Yohkoh and full-disk magnetograms from Kitt Peak, they found the relationship was a power law with an index between 1.6 and 2.0. However, they found a linear relationship for discrete solar features (X-ray bright points and active regions) as for other active stars. The relationships between the radiative and magnetic-flux densities for the Sun-as-a-star have been studied by Preminger et al. (2010). We have not been able to find solar studies comparing full-disk chromospheric flux to full-disk photospheric magnetic flux. Livingston et al. (2007) discussed the spectrum variability of the Sun-as-a-star and its correlation with the sunspot number. Schrijver \& Harvey (1989) and Harvey \& White (1999) found that average Ca II K-line flux density is proportional to the average magnetic-flux density to the power 0.6 , when averaging over non-sunspot chromospheric regions. Ortiz \& Rast (2005) obtained a similar result when comparing Ca II K-line intensity with magnetic-flux density for image sections near the disk centre. Also, Schrijver (1983) showed that the average solar coronal X-ray flux depends on the average chromospheric flux to the power $\approx 1.6$.

For more than three decades, the total solar irradiance (TSI) has been monitored from several radiometers from space (e.g. Foukal \& Lean 1988; Fröhlich et al. 1995; Kariyappa \& Pap 1996; Rottman et al. 2006). Recent space instrumentation and improved understanding of instrument internal stray light contributions (Schmutz et al. 2013) has led to more accurate values for the TSI and derived parameters (e.g. Kopp \& Lean 2011; Prša et al. 2016). It was observed that the solar energy flux changes over a solar cycle. It has been shown that the solar cycle irradiance variations are attributed to the changes in total brightness and area of the magnetic elements (Foukal \& Lean 1988; Kariyappa \& Pap 1996; Worden et al. 1998; Kariyappa 2000, 2008; Veselovsky et al. 2001; Kumara et al. 2012; Yeo et al. 2014b) whereas the short-term irradiance variations are directly associated with active regions as they evolve and move across the solar disk (Lean 1987). To understand and clarify the difference between the observed and modelled irradiance variability, know the underlying physical mechanisms of solar irradiance variability, and determine the role of various coronal features and corresponding photospheric magnetic features in the EUV and UV irradiance, a detailed and accurate image analysis of spatially resolved observations of the intensity images and magnetograms is required. To achieve this task, a successful segmentation of the solar features is necessary. The image segmentation is an important tool in the reconstruction of solar spectral irradiance (SSI) in the line of the semi-empirical reconstruction approaches (Fontenla et al. 2009; Haberreiter 2011; Ball et al. 2011; Haberreiter et al. 2014; Fontenla et al. 2017).

Our objective in this study is the practical conversion of solar intensity and magnetic field images into physically interpretable quantities describing the solar magnetic cycle and its consequences on the radiative output of the Sun. We have used the Spatial Possibilistic Clustering Algorithm (SPoCA; Barra et al. 2008, 2009; Verbeeck et al. 2014; Kumara et al. 2014), which is an image segmentation algorithm that allows the separation of solar intensity images and magnetograms into three characteristic structures on the Sun: active regions (ARs), coronal holes (CHs), and quiet sun (QS) (Kumara et al. 2014). Understanding the EUV and UV irradiance variability from spatially resolved intensity and magnetic field observations is an important issue in space weather and climate applications.

\section{Observations and analysis}

\subsection{Observations}

We used data from the Atmospheric Imaging Assembly (AIA; Boerner et al. 2012) and Helioseismic and Magnetic Imager (HMI; Hoeksema et al. 2014) instruments on board the Solar Dynamics Observatory (SDO; Pesnell et al. 2012) and the Large Yield Radiometer (LYRA; Hochedez et al. 2006; Dominique et al. 2013) and the Sun Watcher with Active Pixel System detector and Image Processing telescope (SWAP; Berghmans et al. 2006; Seaton et al. 2013), both on board the PROBA2 spacecraft. We have considered Level 1.5 AIA (171 A, $193 \AA$ ) and Level 1 SWAP (174 ̊) EUV images and HMI Level 1.5, full-disk line-of-sight (LOS) magnetograms. The SWAP and AIA images are corrected for dark currents, are flatfield corrected, bad pixels are removed, and the images are rotated such that the solar north is at the top. The SWAP imager experiences a detector degradation of less than $2 \%$ per year (private communication with the SWAP Principal Investigator), which may result in the increase of dark currents. These effects are also corrected for in the calibrated data. The data from the AIA imager was further cross-calibrated to the EVE calibration rocket under flight, which resulted in a good agreement of both. Further calibration rocket flights will allow an update of the AIA instrument spectral calibration curve (Boerner et al. 2012). The HMI LOS magnetograms are fully corrected for detector, optics, and degradation effects; see Couvidat et al. (2016) for a full description. The level 3 data of the aluminium filter channel of the Large Yield Radiometer (LYRA) was used to compare against the solar irradiance over the full time period. The LYRA time series are fully radiometrically calibrated and then averaged over one minute. The detector is responsive to wavelengths from $17 \mathrm{~nm}-80 \mathrm{~nm}$ plus a contribution below $5 \mathrm{~nm}$, including the strong He II at $30.4 \mathrm{~nm}$ (Dominique et al. 2013).

\subsection{Analysis}

In our previous paper, Kumara et al. (2014), we described the details of all the instruments, calibration of data, degradation 
of instruments, and the SPoCA. We also explained the observational details and procedure applied to segment the coronal features (ARs/CHs/QS/FD) for the period January 2011 to December 2012. In this paper we used the segmented maps of ARs/CHs/QS/FD from the previous analysis for $2 \mathrm{yr}$ (2011 and 2012) and extended similar analysis up to January 2016. The time series are created by the following steps:

- Data selection;

- Segmentation of EUV images;

- Creation of AR/CH/QS maps;

- Extending the AR maps into extended active regions (eAR) maps;

- Applying these maps on all EUV channels and HMI magnetograms;

- Creation of time series for eAR/CH/QS for each EUV channel and HMI magnetogram by integration over all pixels of a coronal feature.

The maps have been projected/overlaid onto the HMI full-disk LOS magnetograms to segment the photospheric magnetic features corresponding to coronal $\mathrm{ARs} / \mathrm{CHs} / \mathrm{QS}$ regions for the period of five years. Our analysis also includes the integrated intensity over the full disk (FD).

For the period of five years, starting from January 1, 2011, we selected the HMI magnetograms that are nearest in time to the available AIA segmentation maps. In order to study the longterm variations, we selected six equally distributed images per day. The segmentation maps are arrays with the same dimension as the input images; these are $4 \mathrm{k} \times 4 \mathrm{k}$ arrays in the case of AIA and $1 \mathrm{k} \times 1 \mathrm{k}$ arrays in the case of SWAP. In order to apply the segmentation maps on HMI magnetograms, it is necessary to first match the image scale between the different instruments. To achieve this, we rescaled the SPoCA maps by taking the ratio of the solar disk radii from AIA and HMI observations as a scaling parameter. Furthermore, we aligned the AIA maps to achieve spatial alignment between the SWAP maps, AIA maps, and HMI magnetograms. The full resolution AIA segmentation maps were used on AIA images to compute the integrated intensity values over ARs/CHs/QS/FD. We then rebinned these segmentation maps and the AIA and SWAP images to align with HMI magnetograms. The pixels of the map can have either zero or non-zero values. Non-zero values correspond to the pixels located in an ARmap or CHmap. Since maps and images have the same size, they share the same coordinate system. For example, all non-zero pixels in an ARmap have the same coordinates as all the pixels inside active regions in the corresponding AIA image. It is easy to extract these coordinates with the WHERE command in IDL. Therefore we can obtain the coordinates of all pixels inside ARs and $\mathrm{CHs}$ using ARmaps and CHmaps.

After applying the ARmaps onto the LOS magnetograms, each individual active region is further expanded into its neighbourhood pixels. By careful visual inspection of many active region areas, we found that this extension would improve the overall segmentation. For coronal hole regions, a further adaptation of the segmented region was found to be of marginal value. The effects due to the different acquisition time between the individual channels and observations of magnetograms and EUV images at different altitudes are not considered in our analysis. The extended magnetic regions are indicated as extended active regions, eAR, in the following section.

The mean value of the magnetic flux within a month of $A R_{\mathrm{HMI}}$ is, for example for January 2012, 49 Gauss and the standard deviation is 20 Gauss. The monthly mean and standard deviation of the $e A R_{\mathrm{HMI}}$ is 70 and 19 Gauss, respectively. For

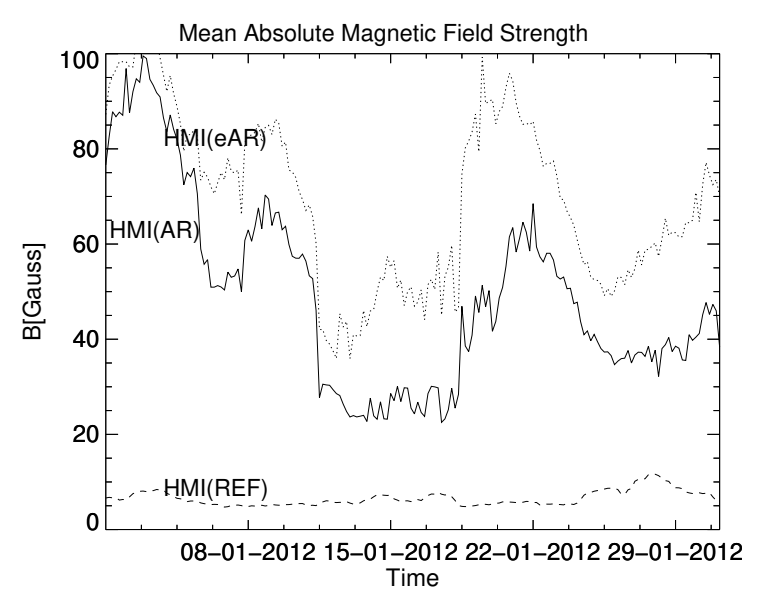

Fig. 1. Mean values of the absolute magnetic field of the ARs, eARs, and a reference region for January 2012. The solid line represents the mean value obtained for each image of the absolute values of the magnetic field within an active region, the dotted line represents the corresponding values of the extended active region, and the dashed line represents the values of a reference region.

comparison, we selected a reference region in the centre of the solar disk (indicated as $R E F$ in Fig. 1). The region is the same size, i.e. the same amount of pixels, but does not include any active region nor coronal hole pixels. The region has a mean value of 6.5 and a standard deviation of 1.5 Gauss. Figure 1 shows the evolution of the daily mean values described above for the month of January 2012.

Our algorithm extends the $A R_{\mathrm{HMI}}$ into an $e A R_{\mathrm{HMI}}$ in the following way: let $e A R_{\mathrm{HMI}}$ be $A R_{\mathrm{HMI}}$. For each adjacent pixel outside of $e A R_{\mathrm{HMI}}$ check if its value is above a threshold $T$ and expand $e A R_{\mathrm{HMI}}$ with the selected pixel. The selection is repeated until all adjacent pixels are below the threshold $T$. The $e A R_{\mathrm{HMI}}$ is thus always larger or equal than the original projection $A R_{\mathrm{HMI}}$. The threshold $T$ was set to 35 Gauss, that is larger than the monthly mean value for the QS region (19 Gauss). The $e A R_{\mathrm{HMI}}$ cover typically $20 \%$ more pixels than $A R_{\mathrm{HMI}}$, as can be seen from Fig. 1. We did not find a large correlation value between the mean absolute magnetic field values and the area increase between the $A R_{\mathrm{HMI}}$ and the $e A R_{\mathrm{HMI}}$. The Spearman rank coefficient for this correlation is 0.55 for all data from 2012 .

An illustration of SWAP and HMI maps is shown in Fig. 2. With these coordinates, we can easily extract the pixel values of the ARs and CHs. In the left panel we show the SWAP $17.4 \mathrm{~nm}$ segmented ARs/CHs/QS/FD map. The right panel is the segmented map overlaid on the HMI magnetogram showing the eARs/ARs/CHs/QS/FD. features. In Fig. 3, several cases for the extension algorithm are mapped, covering four examples of the most common active regions classes, as defined by Jaeggli \& Norton (2016). The algorithm works satisfactorily for examples of active region classes $\alpha, \beta, \beta-\gamma$, and $\beta-\gamma-\delta$ classes, as plotted in the individual sub-panels. Especially, in the case of complex active regions, i.e. the $\beta-\gamma-\delta$ case, the eAR is covering the increased magnetic activities. As these classes represent more than $90 \%$ of the active regions (Jaeggli \& Norton 2016), we assume that our mapping algorithm works satisfactorily; see one-month movie attached to Fig .2. Variations in the quantities resulting from the segmentation, such as the integrated intensity and photospheric magnetic flux density of the coronal features and the full-disk observations, are compared with those of the LYRA irradiance and sunspot numbers. To allow a statistical view on the analysis, a regression analysis is performed 

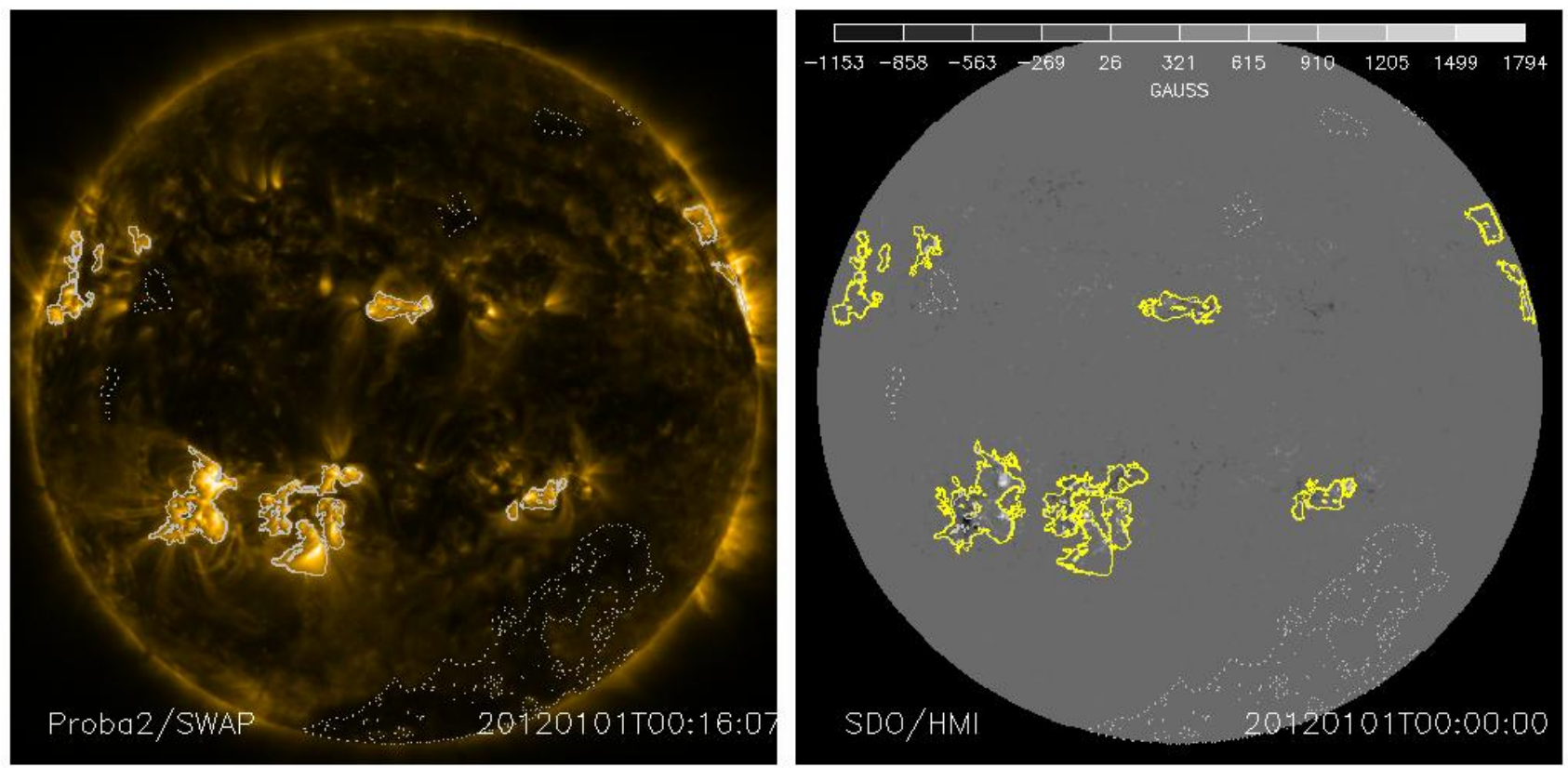

(a) Proba2/SWAP $17.4 \mathrm{~nm}$ and SDO/HMI LOS magnetogram

Fig. 2. Left panel: segmentation maps resulting from the SPoCA algorithm applied on SWAP $17.4 \mathrm{~nm}$ image. The solid grey line indicates the segmented AR. The dotted grey line indicates the segmented CH. Right panel: photospheric magnetic features are identified in the HMI magnetogram using the SPoCA segmentation maps. The grey lines indicate the segmented features projected onto the magnetogram. The yellow line indicates the extended AR area. The temporal evolution over one month is shown by a movie available online.

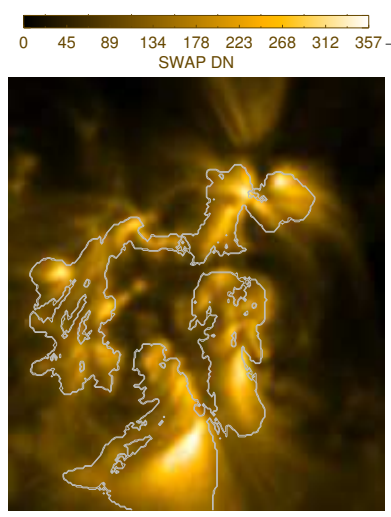

PROBA2/SWAP

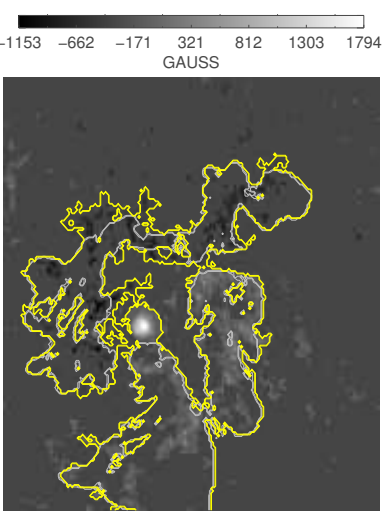

$\mathrm{SDO} / \mathrm{HMI}$ Magnetogram

(a) $\alpha$ magnetic configuration (1 January 2012, 00:18, NOAA 11388)

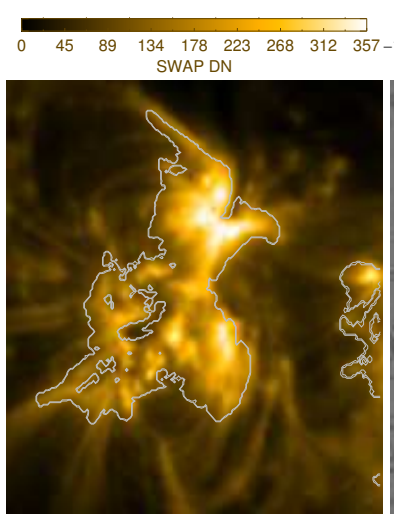

PROBA2/SWAP

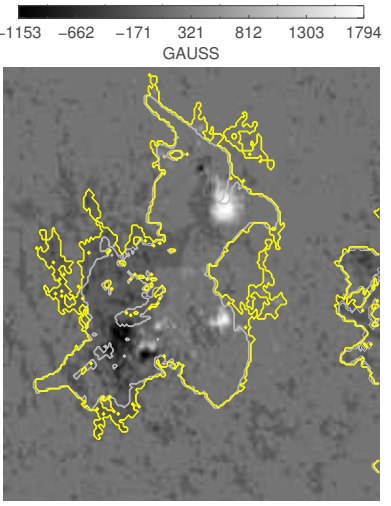

SDO/HMI Magnetogram

(c) $\beta$ - $\gamma$ magnetic configuration (1 January 2012, 00:16, NOAA 11389)

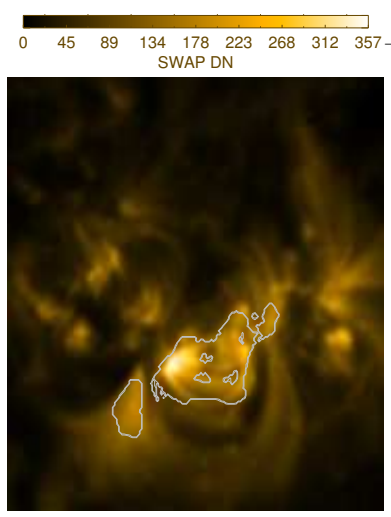

PROBA2/SWAP

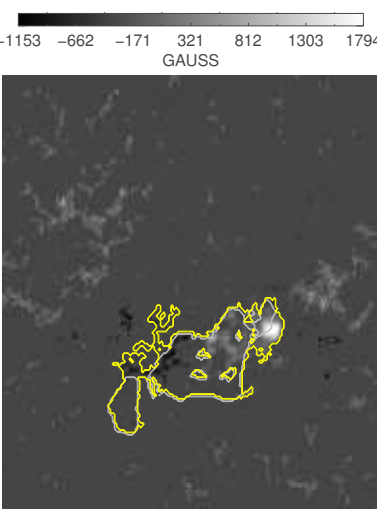

SDO/HMI Magnetogram

(b) $\beta$ magnetic configuration (1 January 2012, 00:16, NOAA 11386)

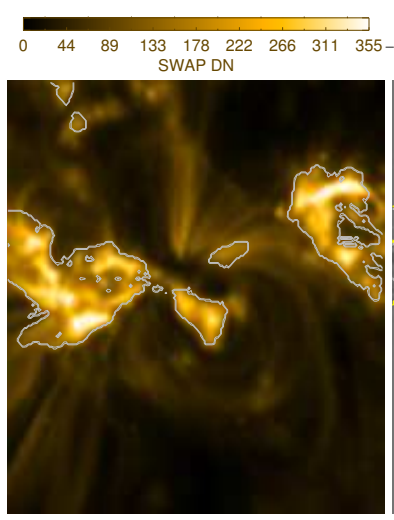

PROBA2/SWAP

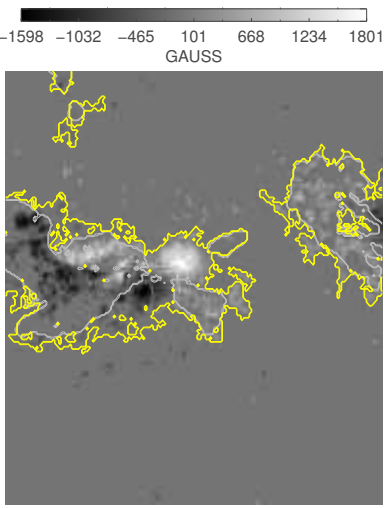

SDO/HMI Magnetogram

(d) $\beta-\gamma-\delta$ magnetic configuration (9 March 2012, 00:15, NOAA 11429)

Fig. 3. Left panels: coronal active region identified with the SPoCA segmentation maps for different magnetic configurations (grey lines). Right panels: photospheric magnetic features identified by the extended active region maps (yellow lines). 


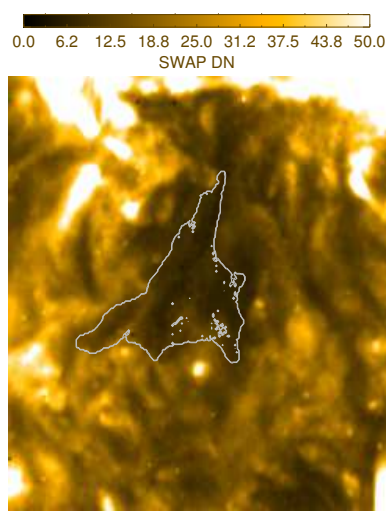

PROBA2/SWAP

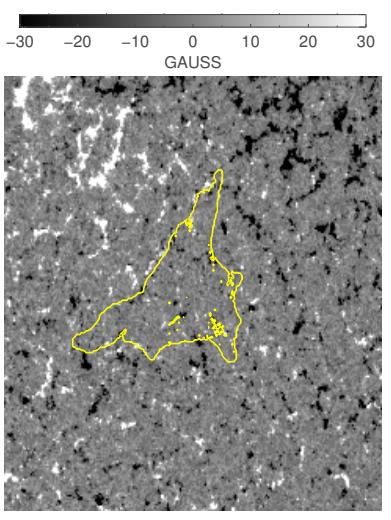

$\mathrm{SDO} / \mathrm{HMI}$ Magnetogram

(a) 21 January 2012, 03:58, CH region

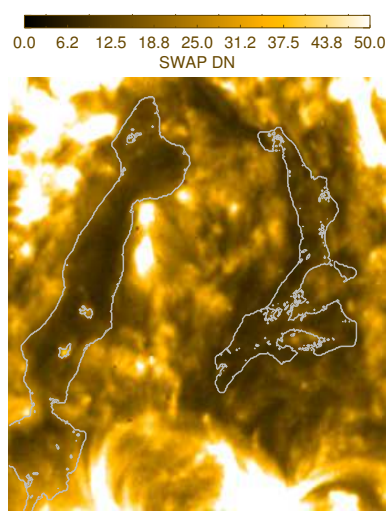

PROBA2/SWAP

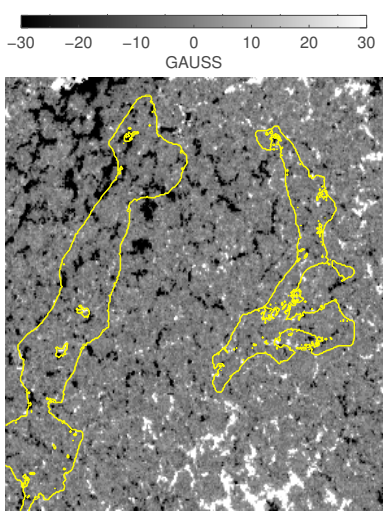

SDO/HMI Magnetogram (b) 3 January 2012, 11:58, CH region

Fig. 4. Coronal hole segmentation map examples overlaid onto the SWAP images (left) and the photospheric magnetic images (right).

by computing a linear fit $y=a+b x$ for all parameters. The confidence interval for the offset $a$ and the slope $b$ is computed, along with the Spearman rank correlation coefficients and its $1 \sigma$ uncertainty, as detailed in Tamhane \& Dunlop (2000).

\section{Results}

The segmented maps derived using the SPoCA algorithm from AIA (17.1 nm, $19.3 \mathrm{~nm}$ ) images for the period of five years (January 2011 to January 2016) were applied to HMI full-disk LOS magnetograms. The SPoCA derived active region maps were extended to all adjacent pixels with a magnetic flux of higher than 35 Gauss before the maps were applied to the HMI images. We computed the integrated magnetic flux density of the full-disk and $\mathrm{eARs} / \mathrm{CHs} / \mathrm{QS} / \mathrm{FD}$ features from the segmented magnetic maps. We then compared these quantities with the SWAP segmented features, the daily-averaged SSI as observed with the LYRA aluminium channel (observed Sun as a star), and to the sunspot numbers. This comparison includes the calculation of the Spearman rank coefficient between all the observables.

\subsection{Integrated intensity and absolute magnetic flux density variations of the segmented coronal features}

In Fig. 5 we plot the time series of total absolute magnetic flux density of each of the coronal features (eARs, CHs, and QS) and full-disk absolute magnetic flux density from the SDO/HMI magnetograms. The LYRA aluminium channel irradiance for the five years is shown in the lower left panel. For comparison, we plotted the sunspot number for the same period in the right bottom panel. Further, Fig. 6 show the time series of feature-wise integrated intensity, the full-disk intensities obtained from the SWAP segmented maps, respectively, and the sunspot numbers for the corresponding period for comparison. Table 1 presents the mean, minimum, maximum, and standard deviation of the integrated intensity of ARs, CHs, QS, and the full disk derived from AIA images, the integrated magnetic flux densities of eARs, CHs, QS, and the full disk derived from HMI magnetograms, and LYRA aluminium channel irradiance measurements for a five-year period.

It can be seen from the figures and Table 1 that the total magnetic flux density of active regions is larger than that of the coronal holes, but smaller than the flux of the quiet sun. The area covered by the quiet sun (QS) regions is larger than ARs and CHs, hence the total absolute magnetic flux density is larger than that of ARs. Table 1 also lists the mean of the expected noise level for each segmented region of the magnetogram data. The noise level is computed as the product of the number of pixels and the detector shot noise, 5 Gauss, as specified in Hoeksema et al. (2014).

Figure 7 represent the scatter plots of integrated intensities over SWAP coronal features against the respective HMI integrated absolute magnetic flux density. The bottom panels represent LYRA aluminium channel irradiance and the sunspot number versus the HMI full-disk average flux density. These scatter plots show that the ARs, CHs, QS, and FD intensities have good correlation with the corresponding absolute magnetic flux density.

Table 2 gives a statistical analysis of all analysed time series. A linear regression analysis, $y=a+b x$, results in the offset $a$ and slope $b$ for a best mean-square linear fit. Further, the Spearman correlation coefficients between HMI, AIA 17.1 m, SWAP $17.4 \mathrm{~nm}$, and the LYRA aluminium channe, for all segmented features (ARs/CHs/QS) and the total integrated full-disk intensity (FD), are given. The second and third column in Table 2 list the linear fitting parameters $a, b$, and the Spearman correlation coefficient $r$, respectively. The next three columns list the $95 \%$ confidence intervals, thus the $1 \sigma$ value, of these fitting parameters: Col. 5 lists the $95 \%$ confidence interval of the parameter $a$, Col. 6 the $95 \%$ confidence interval of the parameter $b$, and Col. 7 the $95 \%$ confidence interval of the correlation parameter $r$ itself. The $1 \sigma$ value of the difference $d$ between the original data and fitted data, for example for the full-disk

$d=F D_{\mathrm{SWAP}}-\left(a+b * F D_{\mathrm{HMI}}\right)$,

is listed in Col. 8. The last column represents the expected noise level $n$ and is computed, for example for the full-disk value, as follows:

$n=F D_{\mathrm{SWAP}}-\left(a+b *\left(F D_{\mathrm{HMI}}+N\left(F D_{\mathrm{HMI}}\right)\right)\right)$

where $N\left(F D_{\mathrm{HMI}}\right)$ is the number of pixels of the solar disk multiplied by the expected noise value (5 Gauss).

The correlation coefficients listed in Table 2, Col. 4, indicate a very good relationship between all the SWAP and HMI time series. Noise levels, as listed in the last column in Table 2, that are smaller than the $1 \sigma$ value of the difference between the original and fitted data give us confidence in the significance of the calculated correlation coefficients. This is the case for most 

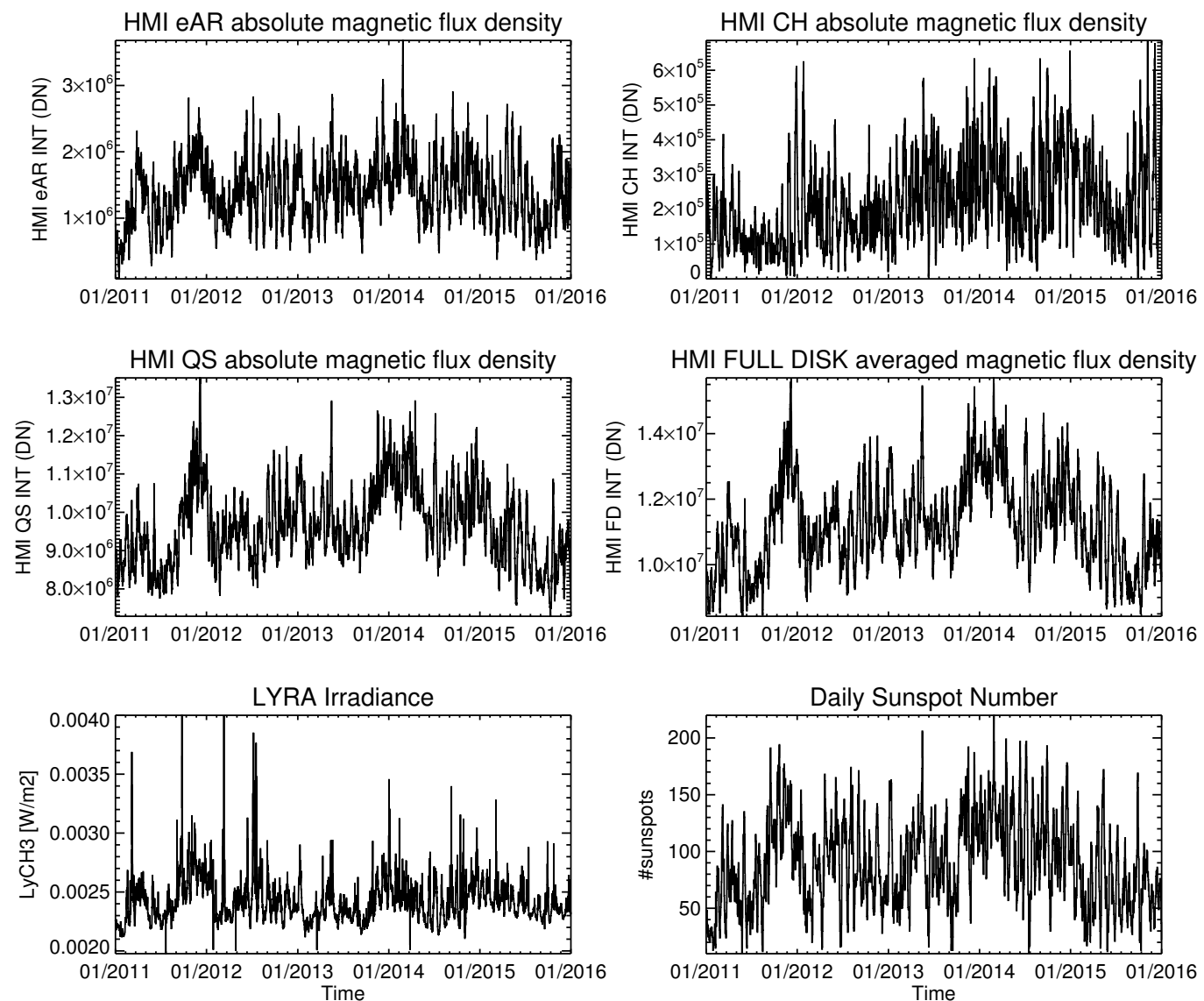

Fig. 5. The time series of total magnetic flux density of eARs, CHs, QS, and full-disk obtained from the HMI magnetograms. The lower left panel shows the time series of LYRA aluminium channel irradiance observations and the lower right panel shows the daily sunspot number variations for comparison. All the quantities are plotted for the period of five years starting from 1 January 2011.
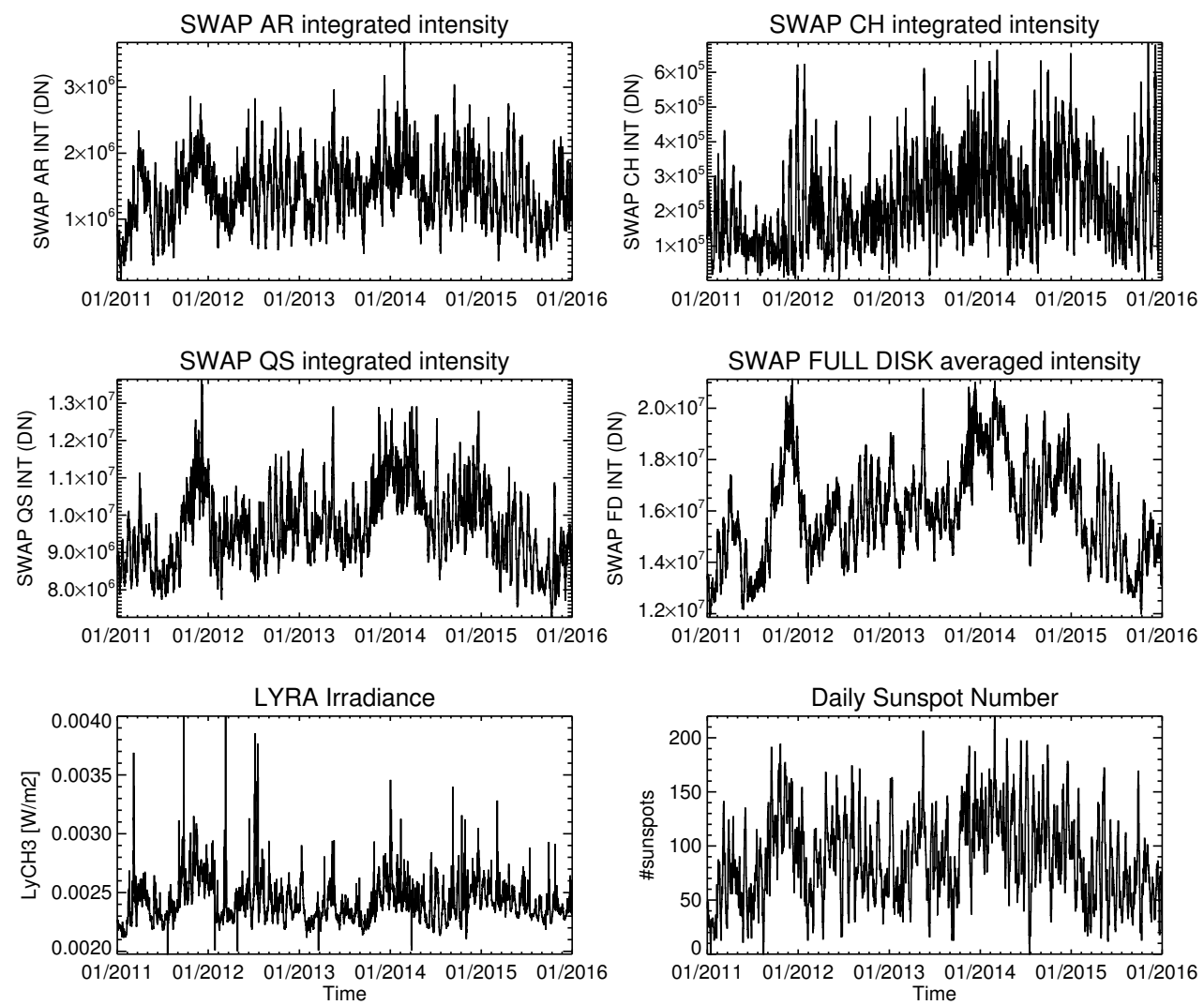

Fig. 6. Same as Fig. 5, but plotted for the SWAP observations. 
Table 1. Mean, minimum, maximum, and standard deviation of the time series for the five-year period on the integrated magnetic field of HMI, the integrated intensity of AIA and SWAP for ARs/CHs/QS/FD, and the LYRA aluminium channel irradiance observations.

\begin{tabular}{cccccc}
\hline \hline & AR & CH & QS & FD & $\begin{array}{c}\text { LYRA AL } \\
\text { DN }\end{array}$ \\
\hline \multicolumn{5}{c}{ For AIA: 1 Jan. 2011-1 Feb. 2016 } \\
\hline Mean & $1.744 \mathrm{E}+08$ & $3.371 \mathrm{E}+07$ & $1.133 \mathrm{E}+09$ & $1.809 \mathrm{E}+09$ & $2.386 \mathrm{E}-03$ \\
Min & $9.859 \mathrm{E}+06$ & $9.573 \mathrm{E}+05$ & $7.576 \mathrm{E}+08$ & $1.284 \mathrm{E}+09$ & $1.000 \mathrm{E}-03$ \\
Max & $4.683 \mathrm{E}+08$ & $3.291 \mathrm{E}+08$ & $1.750 \mathrm{E}+09$ & $2.651 \mathrm{E}+09$ & $4.000 \mathrm{E}-03$ \\
Stddev & $7.104 \mathrm{E}+07$ & $3.155 \mathrm{E}+07$ & $1.446 \mathrm{E}+08$ & $2.309 \mathrm{E}+08$ & $2.047 \mathrm{E}-04$ \\
\hline \multicolumn{6}{c}{ For HMI magnetograms: 1 Jan. 2011-1 Feb. 2016 } \\
\hline Mean & $1.301 \mathrm{E}+06$ & $1.994 \mathrm{E}+05$ & $9.467 \mathrm{E}+06$ & $1.095 \mathrm{E}+07$ \\
Min & $6.128 \mathrm{E}+02$ & $3.052 \mathrm{E}-06$ & $6.140 \mathrm{E}+06$ & $6.638 \mathrm{E}+06$ & \\
Max & $3.679 \mathrm{E}+06$ & $6.858 \mathrm{E}+05$ & $1.351 \mathrm{E}+07$ & $1.570 \mathrm{E}+07$ & \\
Stddev & $5.340 \mathrm{E}+05$ & $1.231 \mathrm{E}+05$ & $1.054 \mathrm{E}+06$ & $1.486 \mathrm{E}+06$ & \\
Noise & $3.769 \mathrm{E}+04$ & $6.021 \mathrm{E}+04$ & $1.352 \mathrm{E}+06$ & $1.450 \mathrm{E}+06$ & \\
\hline \multicolumn{7}{c}{ For SWAP magnetograms: 1 Jan. 2011-1 Feb. 2016 } \\
\hline Mean & $1.317 \mathrm{E}+06$ & $2.018 \mathrm{E}+05$ & $9.495 \mathrm{E}+06$ & $1.557 \mathrm{E}+07$ & \\
Min & $4.442 \mathrm{E}+04$ & $6.864 \mathrm{E}+02$ & $6.894 \mathrm{E}+06$ & $1.061 \mathrm{E}+07$ & \\
Max & $3.679 \mathrm{E}+06$ & $6.858 \mathrm{E}+05$ & $1.364 \mathrm{E}+07$ & $2.113 \mathrm{E}+07$ & \\
Stddev & $5.359 \mathrm{E}+05$ & $1.234 \mathrm{E}+05$ & $1.064 \mathrm{E}+06$ & $2.083 \mathrm{E}+06$ \\
\hline
\end{tabular}

Table 2. Regression statistics of all analysed time series.

\begin{tabular}{ccccccccc}
\hline \hline Full Disk & $a$ & $b$ & $r$ & $\mathrm{CI}(\mathrm{a})$ & $\mathrm{CI}(\mathrm{b})$ & $1-\sigma(r)$ & $1-\sigma(d)$ & Noise \\
\hline HMI vs. SWAP & $1.463 \mathrm{E}+06$ & 1.292 & 0.937 & $1.059 \mathrm{E}+05$ & 0.009379 & 0.004785 & $6.815 \mathrm{E}+05$ & $1.873 \mathrm{E}+06$ \\
HMI vs. AIA & $5.319 \mathrm{E}+08$ & 114.1 & 0.635 & $2.873 \mathrm{E}+07$ & 2.545 & 1.298 & $1.849 \mathrm{E}+08$ & $1.655 \mathrm{E}+08$ \\
HMI vs. LYRA & 0.001758 & $5.816 \mathrm{E}-11$ & 0.574 & $2.184 \mathrm{E}-05$ & $1.935 \mathrm{E}-12$ & $9.869 \mathrm{E}-13$ & 0.0001406 & $8.435 \mathrm{E}-05$ \\
HMI vs. SSN & -152.0 & $2.142 \mathrm{E}-05$ & 0.739 & 4.181 & $3.704 \mathrm{E}-07$ & $1.889 \mathrm{E}-07$ & 26.91 & 31.06 \\
\hline AR & & & & & & & & \\
HMI vs. SWAP & $7.006 \mathrm{E}+04$ & 0.9619 & 0.958 & 7834. & 0.005312 & 0.002710 & $1.371 \mathrm{E}+05$ & $3.625 \mathrm{E}+04$ \\
HMI vs. AIA & $8.807 \mathrm{E}+06$ & 126.5 & 0.926 & $1.479 \mathrm{E}+06$ & 1.003 & 0.5117 & $2.589 \mathrm{E}+07$ & $4.766 \mathrm{E}+06$ \\
HMI vs. LYRA & 0.002184 & $1.619 \mathrm{E}-10$ & 0.569 & $8.060 \mathrm{E}-06$ & $5.465 \mathrm{E}-12$ & $2.788 \mathrm{E}-12$ & 0.0001411 & $6.100 \mathrm{E}-06$ \\
HMI vs. SSN & 11.54 & $5.495 \mathrm{E}-05$ & 0.684 & 1.685 & $1.143 \mathrm{E}-06$ & $5.829 \mathrm{E}-07$ & 29.49 & 2.071 \\
\hline CH & & & & & & & & \\
HMI vs. SWAP & $1.318 \mathrm{E}+04$ & 0.9511 & 0.951 & 1450. & 0.005896 & 0.003008 & 39.49 & 2.422 \\
HMI vs. AIA & $8.088 \mathrm{E}+06$ & 129.7 & 0.889 & $1.085 \mathrm{E}+06$ & 4.413 & 2.251 & $2.857 \mathrm{E}+07$ & $7.810 \mathrm{E}+06$ \\
HMI vs. LYRA & 0.002392 & $8.146 \mathrm{E}-11$ & 0.070 & $6.123 \mathrm{E}-06$ & $2.490 \mathrm{E}-11$ & $1.270 \mathrm{E}-11$ & 0.0001612 & $4.905 \mathrm{E}-06$ \\
HMI vs. SSN & 79.46 & $4.023 \mathrm{E}-05$ & 0.105 & 1.500 & $6.099 \mathrm{E}-06$ & $3.112 \mathrm{E}-06$ & 39.49 & 2.422 \\
\hline QS & & & & & & & & \\
HMI vs. SWAP & $2.931 \mathrm{E}+05$ & 0.9727 & 0.967 & $4.925 \mathrm{E}+04$ & 0.005094 & 0.002599 & $2.746 \mathrm{E}+05$ & $1.315 \mathrm{E}+06$ \\
HMI vs. AIA & $3.125 \mathrm{E}+08$ & 84.06 & 0.532 & $2.151 \mathrm{E}+07$ & 2.225 & 1.135 & $1.199 \mathrm{E}+08$ & $1.137 \mathrm{E}+08$ \\
HMI vs. LYRA & 0.001758 & $6.770 \mathrm{E}-11$ & 0.485 & $2.622 \mathrm{E}-05$ & $2.712 \mathrm{E}-12$ & $1.384 \mathrm{E}-12$ & 0.0001462 & $9.156 \mathrm{E}-05$ \\
HMI vs. SSN & -159.8 & $2.578 \mathrm{E}-05$ & 0.654 & 5.377 & $5.562 \mathrm{E}-07$ & $2.837 \mathrm{E}-07$ & 29.98 & 34.86 \\
\hline
\end{tabular}

of the correlations and indicated by the greyed cells in the last column of Table 2 .

The correlation coefficients between the corresponding HMI and AIA features are high and they are particularly significant for $\mathrm{AR}$ and $\mathrm{CH}$, but a low correlation was found for the full-disk and QS regions. Figure 8 show the scatter plots of integrated intensities over the AIA coronal features against the respective HMI magnetic flux density. Table 3 contains the yearly correlation coefficients and a good and significant correlation between the AIA features and the magnetic flux densities was identified.

As discussed in Raftery et al. (2013), the AIA $17.1 \mathrm{~nm}$ and the SWAP $17.4 \mathrm{~nm}$ bandpasses are considerably different. Whereas the AIA $17.1 \mathrm{~nm}$ bandpass has a sharp response peak at $17.1 \mathrm{~nm}$, the SWAP response is flat between $17.0 \mathrm{~nm}$ and $17.7 \mathrm{~nm}$. These observations and the different spectral instrument response characteristics might indicate that the AIA $17.1 \mathrm{~nm}$ channel shows either a long-term drift, as noted earlier in Kumara et al. (2012) and BenMoussa et al. (2013), or that the QS intensity at $17.1 \mathrm{~nm}$ is decreasing in time. A more detailed study on these observations is needed.

\subsection{Full-disk magnetic field and intensity variations in comparison with LYRA EUV and UV irradiance}

As observed through the scatter plots (Fig. 5) the full-disk intensity of SWAP is correlated well with their corresponding 

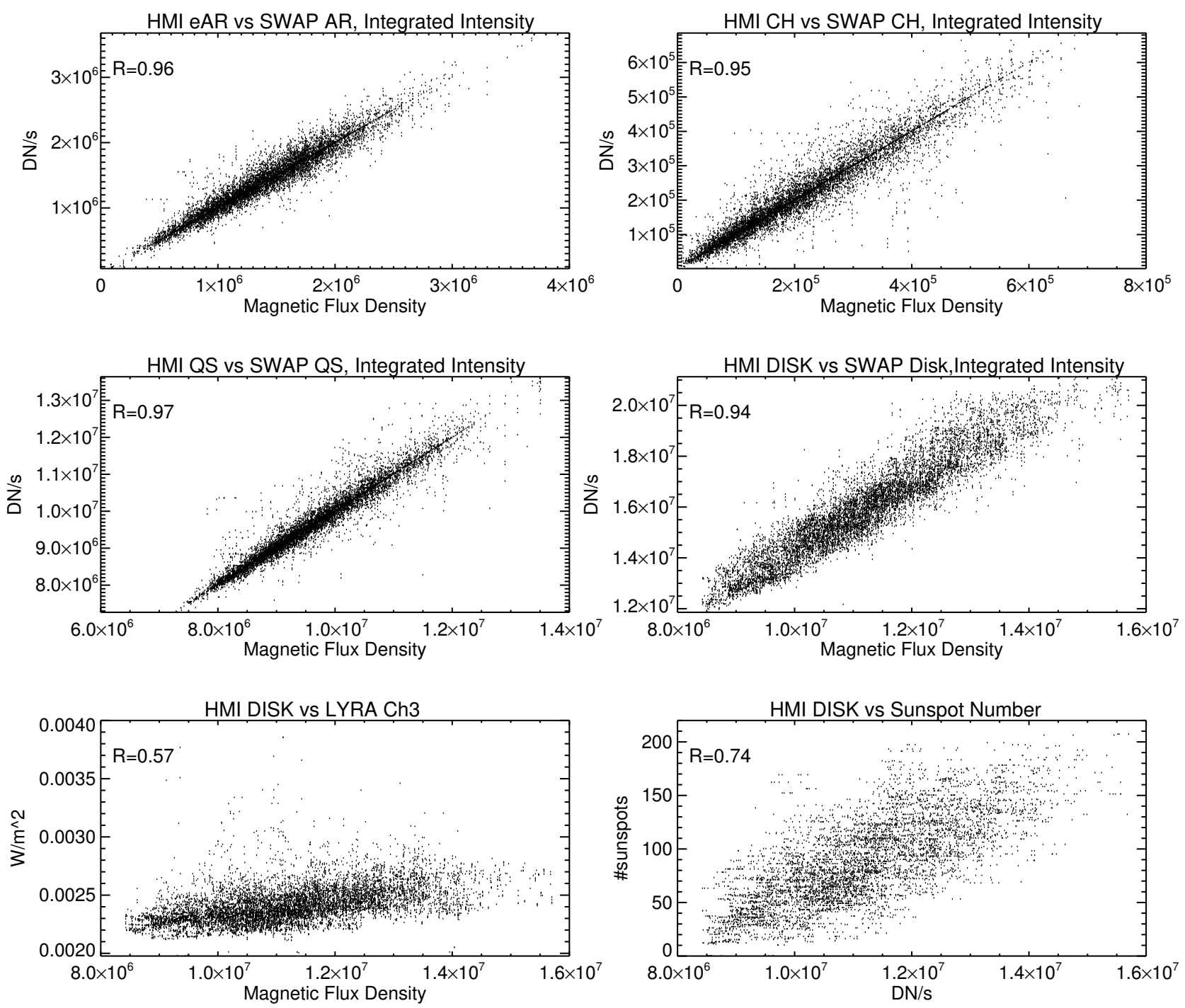

Fig. 7. Scatter plots of integrated intensities over the SWAP coronal features against the respective HMI magnetic flux density. HMI eAR vs., SWAP eAR, HMI CH vs. SWAP CH, HMI QS vs. SWAP QS, and HMI full-disk vs. SWAP full-disk are shown here. The lowest panel shows the HMI full-disk magnetic density vs. the LYRA aluminium channel and vs. the daily sunspot number. The data period spans from 1 January 2011 until 1 January 2016.

full-disk average magnetic field. The intensity of ARs, CHs, and QS measured in both SWAP and AIA experiments has shown a very good correlation with the total magnetic flux density of these features estimated from HMI magnetograms. The relation between the intensity and magnetic field suggests that the magnetic field plays an important role in the heating of these features. Surprisingly, we do not confirm a good correlation between the LYRA aluminium channel and the SWAP full-disk signal $(r=0.57)$, as indicated in Yalim \& Poedts (2014). The computation of the correlation coefficients between the HMI features and the sun-as-a-star LYRA signal results in similar low values, i.e. $0.57,0.48,0.07$, and 0.57 for $e A R_{\mathrm{HMI}}, Q S_{\mathrm{HMI}}, C H_{\mathrm{HMI}}$, and $F D_{\text {HMI }}$ correspondingly, analysed over the full data period. The correlation coefficients, especially for the full-disk component computed on a yearly basis, show slightly higher values, as can be noted in Table 4. Also in the case of LYRA aluminium channel data, a long-term drift of the instrument responsivity might be the cause of the obtained results. The correlation coefficients of the yearly analysis indicates that the magnetic field variations are the main cause for fluctuations seen in the LYRA irradiance variations.

\section{Discussion and conclusions}

We compute the total magnetic flux density of eARs/CHs/QS/FD from the segmented magnetic maps (SDO/HMI). These parameters are compared with LYRA irradiance observations measured in the LYRA aluminium channel for the period of five years starting from 1 January 2011. Time-series plots (Figs. 5 and 6) and the scatter plots (Figs. 7 and 8) show that the long-term variability of segmented features, full-disk intensity, and full-disk magnetic flux density have shown good correlation with the EUV observations from SWAP and AIA. The correlation coefficients from both AIA and LYRA are lower, which could be an effect of instrument ageing. Correlation analysis on smaller timescales shows a clearly higher correlation between the solar features. This shows that the coronal emission features obtained in the EUV passband are related with the strength of the magnetic field associated with eARs, $\mathrm{CHs}$, and QS regions.

In addition the good correlation of full-disk intensity of SWAP $(r=0.94)$ with the full-disk absolute magnetic flux density (HMI) suggests that the magnetic field play an important role in the EUV and UV irradiance variations. 
Table 3. Regression statistics applied to the yearly AIA time series. Statistical parameters are the same as in Table 2.

\begin{tabular}{ccccccccc}
\hline \hline FD (HMI vs. AIA) & $a$ & $b$ & $r$ & $\mathrm{CI}(\mathrm{a})$ & $\mathrm{CI}(\mathrm{b})$ & $1-\sigma(r)$ & $1-\sigma(d)$ & Noise \\
\hline 2011 & $4.660 \mathrm{E}+08$ & 147.429 & 0.936 & $2.626 \mathrm{E}+07$ & 2.424 & 1.236 & $8.777 \mathrm{E}+07$ & $4.276 \mathrm{E}+07$ \\
2012 & $6.669 \mathrm{E}+08$ & 108.107 & 0.815 & $3.632 \mathrm{E}+07$ & 3.280 & 1.672 & $7.467 \mathrm{E}+07$ & $3.136 \mathrm{E}+07$ \\
2013 & $2.958 \mathrm{E}+08$ & 128.772 & 0.873 & $3.233 \mathrm{E}+07$ & 2.766 & 1.410 & $7.470 \mathrm{E}+07$ & $3.735 \mathrm{E}+07$ \\
2014 & $3.786 \mathrm{E}+08$ & 120.193 & 0.888 & $3.224 \mathrm{E}+07$ & 2.622 & 1.337 & $7.361 \mathrm{E}+07$ & $3.486 \mathrm{E}+07$ \\
2015 & $3.204 \mathrm{E}+08$ & 115.625 & 0.897 & $2.264 \mathrm{E}+07$ & 2.138 & 1.090 & $5.755 \mathrm{E}+07$ & $3.354 \mathrm{E}+07$ \\
\hline AR (HMI vs. AIA) & & & & & & & & \\
2011 & $4.284 \mathrm{E}+06$ & 150.839 & 0.968 & $2.401 \mathrm{E}+06$ & 1.734 & 0.884 & $2.167 \mathrm{E}+07$ & $1.137 \mathrm{E}+06$ \\
2012 & $1.160 \mathrm{E}+07$ & 131.368 & 0.942 & $2.745 \mathrm{E}+06$ & 1.927 & 0.983 & $1.964 \mathrm{E}+07$ & $9.902 \mathrm{E}+05$ \\
2013 & $1.228 \mathrm{E}+07$ & 120.382 & 0.927 & $2.952 \mathrm{E}+06$ & 1.911 & 0.974 & $2.032 \mathrm{E}+07$ & $9.074 \mathrm{E}+05$ \\
2014 & $1.241 \mathrm{E}+07$ & 117.875 & 0.919 & $3.226 \mathrm{E}+06$ & 1.934 & 0.986 & $2.118 \mathrm{E}+07$ & $8.885 \mathrm{E}+05$ \\
2015 & $-1.629 \mathrm{E}+06$ & 120.051 & 0.973 & $1.542 \mathrm{E}+06$ & 1.148 & 0.586 & $1.260 \mathrm{E}+07$ & $9.049 \mathrm{E}+05$ \\
\hline CH (HMI vs. AIA) & & & & & & & & \\
2011 & $1.155 \mathrm{E}+06$ & 181.830 & 0.948 & $3.603 \mathrm{E}+05$ & 2.180 & 1.112 & $4.831 \mathrm{E}+06$ & $2.190 \mathrm{E}+06$ \\
2012 & $-1.103 \mathrm{E}+06$ & 159.850 & 0.918 & $5.451 \mathrm{E}+05$ & 2.779 & 1.417 & $5.956 \mathrm{E}+06$ & $1.925 \mathrm{E}+06$ \\
2013 & $3.235 \mathrm{E}+06$ & 123.173 & 0.908 & $6.481 \mathrm{E}+05$ & 2.398 & 1.223 & $6.081 \mathrm{E}+06$ & $1.483 \mathrm{E}+06$ \\
2014 & $2.976 \mathrm{E}+06$ & 127.241 & 0.925 & $6.874 \mathrm{E}+05$ & 2.258 & 1.151 & $6.980 \mathrm{E}+06$ & $1.532 \mathrm{E}+06$ \\
2015 & $3.240 \mathrm{E}+07$ & 95.617 & 0.712 & $5.481 \mathrm{E}+06$ & 20.825 & 10.619 & $5.992 \mathrm{E}+07$ & $1.151 \mathrm{E}+06$ \\
\hline QS (HMI vs. AIA) & & & & & & & & \\
2011 & $3.493 \mathrm{E}+08$ & 101.561 & 0.913 & $1.830 \mathrm{E}+07$ & 1.951 & 0.995 & $5.129 \mathrm{E}+07$ & $2.747 \mathrm{E}+07$ \\
2012 & $2.559 \mathrm{E}+08$ & 94.980 & 0.827 & $2.556 \mathrm{E}+07$ & 2.678 & 1.366 & $4.516 \mathrm{E}+07$ & $2.569 \mathrm{E}+07$ \\
2013 & $7.767 \mathrm{E}+07$ & 102.560 & 0.926 & $1.876 \mathrm{E}+07$ & 1.884 & 0.960 & $3.805 \mathrm{E}+07$ & $2.774 \mathrm{E}+07$ \\
2014 & $1.328 \mathrm{E}+08$ & 94.622 & 0.928 & $1.834 \mathrm{E}+07$ & 1.762 & 0.898 & $3.621 \mathrm{E}+07$ & $2.559 \mathrm{E}+07$ \\
2015 & $1.147 \mathrm{E}+08$ & 93.395 & 0.949 & $1.183 \mathrm{E}+07$ & 1.300 & 0.663 & $2.610 \mathrm{E}+07$ & $2.526 \mathrm{E}+07$ \\
\hline
\end{tabular}

Table 4. Regression statistics applied to the yearly LYRA time series. Statistical parameters are the same as in Table 2.

\begin{tabular}{|c|c|c|c|c|c|c|c|c|}
\hline FD (HMI vs. LYRA) & $a$ & $b$ & $r$ & $\mathrm{CI}(\mathrm{a})$ & $\mathrm{CI}(\mathrm{b})$ & $1-\sigma(r)$ & $1-\sigma(d)$ & Noise \\
\hline 2011 & 0.001382 & 9.877E-11 & 0.807 & $4.515 \mathrm{E}-05$ & $4.168 \mathrm{E}-12$ & $2.126 \mathrm{E}-12$ & 0.0001509 & $2.865 \mathrm{E}-05$ \\
\hline 2012 & 0.001821 & $5.476 \mathrm{E}-11$ & 0.499 & 7.906E-05 & $7.139 \mathrm{E}-12$ & $3.640 \mathrm{E}-12$ & 0.0001625 & $1.588 \mathrm{E}-05$ \\
\hline 2013 & 0.001336 & $8.830 \mathrm{E}-11$ & 0.724 & $4.032 \mathrm{E}-05$ & $3.449 \mathrm{E}-12$ & $1.759 \mathrm{E}-12$ & $9.316 \mathrm{E}-05$ & $2.561 \mathrm{E}-05$ \\
\hline 2014 & 0.001648 & $6.357 \mathrm{E}-11$ & 0.510 & $5.932 \mathrm{E}-05$ & $4.826 \mathrm{E}-12$ & $2.461 \mathrm{E}-12$ & 0.0001355 & $1.844 \mathrm{E}-05$ \\
\hline 2015 & 0.001869 & $5.042 \mathrm{E}-11$ & 0.623 & $3.216 \mathrm{E}-05$ & $3.037 \mathrm{E}-12$ & $1.549 \mathrm{E}-12$ & $8.175 \mathrm{E}-05$ & $1.463 \mathrm{E}-05$ \\
\hline \multicolumn{9}{|l|}{ AR (HMI vs. LYRA) } \\
\hline 2011 & 0.002088 & $2.758 \mathrm{E}-10$ & 0.794 & $1.731 \mathrm{E}-05$ & $1.250 \mathrm{E}-11$ & $6.374 \mathrm{E}-12$ & 0.0001562 & $2.079 \mathrm{E}-06$ \\
\hline 2012 & 0.002177 & $1.831 \mathrm{E}-10$ & 0.578 & $2.116 \mathrm{E}-05$ & $1.486 \mathrm{E}-11$ & $7.577 \mathrm{E}-12$ & 0.0001514 & $1.380 \mathrm{E}-06$ \\
\hline 2013 & 0.002066 & $2.012 \mathrm{E}-10$ & 0.693 & $1.502 \mathrm{E}-05$ & $9.720 \mathrm{E}-12$ & 4.956E-12 & 0.0001034 & $1.517 \mathrm{E}-06$ \\
\hline 2014 & 0.002228 & $1.233 \mathrm{E}-10$ & 0.430 & $2.196 \mathrm{E}-05$ & $1.317 \mathrm{E}-11$ & $6.714 \mathrm{E}-12$ & 0.0001442 & $9.293 \mathrm{E}-07$ \\
\hline 2015 & 0.002304 & $7.645 \mathrm{E}-11$ & 0.403 & $1.144 \mathrm{E}-05$ & $8.519 \mathrm{E}-12$ & $4.344 \mathrm{E}-12$ & $9.348 \mathrm{E}-05$ & $5.762 \mathrm{E}-07$ \\
\hline \multicolumn{9}{|l|}{ CH (HMI vs. LYRA) } \\
\hline 2011 & 0.002424 & $1.277 \mathrm{E}-10$ & -0.106 & $1.595 \mathrm{E}-05$ & $9.650 \mathrm{E}-11$ & $4.921 \mathrm{E}-11$ & 0.0002138 & $1.538 \mathrm{E}-06$ \\
\hline 2012 & 0.002433 & $-4.195 \mathrm{E}-11$ & -0.075 & $1.564 \mathrm{E}-05$ & 7.976E-11 & $4.067 \mathrm{E}-11$ & 0.0001709 & $-5.052 \mathrm{E}-07$ \\
\hline 2013 & 0.002274 & $3.576 \mathrm{E}-10$ & 0.219 & $1.409 \mathrm{E}-05$ & 5.213E-11 & $2.658 \mathrm{E}-11$ & 0.0001322 & $4.306 \mathrm{E}-06$ \\
\hline 2014 & 0.002327 & $3.577 \mathrm{E}-10$ & 0.351 & $1.457 \mathrm{E}-05$ & $4.785 \mathrm{E}-11$ & $2.440 \mathrm{E}-11$ & 0.0001479 & $4.308 \mathrm{E}-06$ \\
\hline 2015 & 0.002416 & $-6.652 \mathrm{E}-11$ & -0.028 & $9.120 \mathrm{E}-06$ & $3.465 \mathrm{E}-11$ & $1.767 \mathrm{E}-11$ & $9.971 \mathrm{E}-05$ & $-8.010 \mathrm{E}-07$ \\
\hline \multicolumn{9}{|l|}{ QS (HMI vs. LYRA) } \\
\hline 2011 & 0.001284 & $1.242 \mathrm{E}-10$ & 0.714 & $5.822 \mathrm{E}-05$ & $6.206 \mathrm{E}-12$ & $3.164 \mathrm{E}-12$ & 0.0001631 & $3.359 \mathrm{E}-05$ \\
\hline 2012 & 0.002096 & $3.466 \mathrm{E}-11$ & 0.306 & $9.574 \mathrm{E}-05$ & $1.003 \mathrm{E}-11$ & $5.116 \mathrm{E}-12$ & 0.0001692 & $9.373 \mathrm{E}-06$ \\
\hline 2013 & 0.001400 & $9.701 \mathrm{E}-11$ & 0.552 & $5.405 \mathrm{E}-05$ & $5.427 \mathrm{E}-12$ & $2.768 \mathrm{E}-12$ & 0.0001096 & $2.624 \mathrm{E}-05$ \\
\hline 2014 & 0.001632 & $7.646 \mathrm{E}-11$ & 0.431 & 7.097E-05 & $6.818 \mathrm{E}-12$ & $3.476 \mathrm{E}-12$ & 0.0001401 & $2.068 \mathrm{E}-05$ \\
\hline 2015 & 0.001772 & $6.928 \mathrm{E}-11$ & 0.616 & $3.658 \mathrm{E}-05$ & $4.018 \mathrm{E}-12$ & $2.049 \mathrm{E}-12$ & $8.071 \mathrm{E}-05$ & $1.874 \mathrm{E}-05$ \\
\hline
\end{tabular}

The correlation analysis between LYRA and the full-disk magnetic flux density does not show a strong correlation. When executing the analysis for yearly periods, a high correlation is found for $2011(r=0.80)$ and for $2013(r=0.72)$, which indicates that the variation in the disk-integrated magnetic field modulates the variations of the spectral irradiance as observed with the LYRA aluminium channel. Further investigations with alternative instrumentation is needed to achieve a conclusive result.

In this work we discussed in detail the feasibility and usage of EUV observations to measure irradiance fluctuations. The observations from AIA allow us to segment the images into different coronal features and to compare the corresponding 

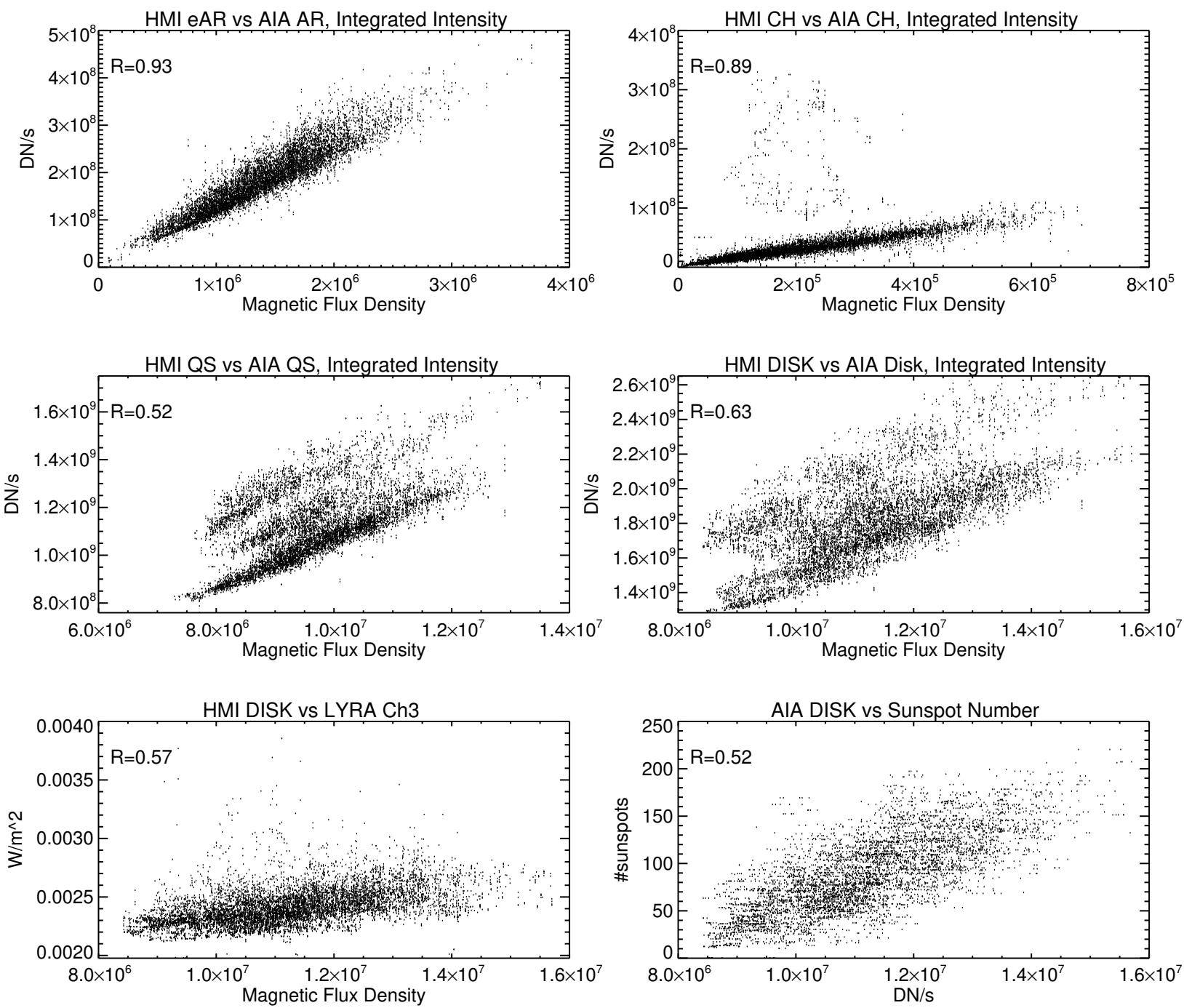

Fig. 8. Scatter plots of integrated intensities over the AIA coronal features against the respective HMI magnetic flux density, as in Fig. 7.

photospheric LOS magnetic field derived from HMI magnetograms. This study also shows that the automated identification and segmentation of the solar images and magnetograms are very useful in reconstructing the SSI variations.

Acknowledgements. The authors would like to thank the PROBA2 science centre, located at the Royal Observatory of Belgium (ROB) in Brussels for providing LYRA and SWAP data taken from January 2011 to January 2016. The sunspot numbers were downloaded from SIDC-SILSO, Royal Observatory of Belgium, Brussels. We also thank Solar Dynamic Observatory (SDO) of National Aeronautics and Space Administration (NASA) for providing AIA and HMI data taken from January 2011 to January 2016. A part of this research work was done at the Indian Institute of Astrophysics when R. Kariyappa (RK) was in regular service. J.J.Z. and R.K. would like to express their sincere thanks to Dr. B.G. Vijayasimha Reddy and Dr. T. Yella Reddy for their encouragement and support on this research. Finally, the authors would like to thank the Faculty of ESA's Science Support Office for the financial support provided. The authors like to thank the reviewer for the constructive comments and suggestions that improved the manuscript.

\section{References}

Babcock, H. W., \& Babcock, H. D. 1955, ApJ, 121, 349

Ball, W. T., Unruh, Y. C., Krivova, N. A., Solanki, S., \& Harder, J. W. 2011, A\&A, 530, A71

Barra, V., Delouille, V., \& Hochedez, J.-F. 2008, Adv. Space Res., 42, 917
Barra, V., Delouille, V., Kretzschmar, M., \& Hochedez, J.-F. 2009, A\&A, 505, 361

BenMoussa, A., Gissot, S., Schühle, U., et al. 2013, Sol. Phys., 288, 389 Berghmans, D., Hochedez, J. F., Defise, J. M., et al. 2006, Adv. Space Res., 38, 1807

Bertello, L., Pevtsov, A., Tlatov, A., \& Singh, J. 2016, Sol. Phys., 291, 2967

Boerner, P., Edwards, C., Lemen, J., et al. 2012, Sol. Phys., 275, 41

Couvidat, S., Schou, J., Hoeksema, J. T., et al. 2016, Sol. Phys., 291, 1887

Dominique, M., Hochedez, J.-F., Schmutz, W., et al. 2013, Sol. Phys., 286, 21

Ermolli, I., Solanki, S. K., Tlatov, A. G., et al. 2009, ApJ, 698, 1000

Ermolli, I., Shibasaki, K., Tlatov, A., \& van Driel-Gesztelyi, L. 2014 Space Sci. Rev., 186, 105

Fontenla, J. M., Curdt, W., Haberreiter, M., Harder, J., \& Tian, H. 2009, ApJ, 707,482

Fontenla, J. M., Codrescu, M., Fedrizzi, M., et al. 2017, ApJ, 834, 54

Foukal, P., \& Lean, J. 1988, ApJ, 328, 347

Fröhlich, C., Romero, J., Roth, H., et al. 1995, Sol. Phys., 162, 101

Haberreiter, M. 2011, Sol. Phys., 274, 473

Haberreiter, M., Delouille, V., Mampaey, B., et al. 2014, J. Space Weather Space Clim., 4, A30

Harvey, K. L., \& White, O. R. 1999, J. Geophys. Res., 104, 19759

Hochedez, J.-F., Schmutz, W., Stockman, Y., et al. 2006, Adv. Space Res., 37, 303

Hoeksema, J. T., Liu, Y., Hayashi, K., et al. 2014, Sol. Phys., 289, 3483

Howard, R. 1959, ApJ, 130, 193

Jaeggli, S. A., \& Norton, A. A. 2016, ApJ, 820, L11

Kariyappa, R. 2000, JA\&A, 21, 293

Kariyappa, R. 2008, JA\&A, 29, 159

Kariyappa, R., \& Pap, J. M. 1996, Sol. Phys., 167, 115

Klimchuk, J. A. 2006, IAU Joint Discussion, 3, 57 


\section{J. J. Zender et al.: Segmentation}

Kopp, G., \& Lean, J. L. 2011, Geophys. Res. Lett., 38, L01706

Kumara, S. T., Kariyappa, R., Dominique, M., et al. 2012, Adv. Astron., 2012, 5

Kumara, S. T., Kariyappa, R., Zender, J. J., et al. 2014, A\&A, 561, A9

Lean, J. 1987, J. Geophys. Res., 92, 839

Leighton, R. B. 1959, ApJ, 130, 366

Livingston, W., Wallace, L., White, O. R., \& Giampapa, M. S. 2007, ApJ, 657, 1137

Ortiz, A., \& Rast, M. 2005, Mem. Soc. Astron. Ital., 76, 1018

Pesnell, W. D., Thompson, B. J., \& Chamberlin, P. C. 2012, Sol. Phys., 275, 3

Pevtsov, A. A., Fisher, G. H., Acton, L. W., et al. 2003, ApJ, 598, 1387

Preminger, D., Nandy, D., Chapman, G., \& Martens, P. C. H. 2010, Sol. Phys., 264, 13

Prša, A., Harmanec, P., Torres, G., et al. 2016, AJ, 152, 41

Raftery, C. L., Bloomfield, D. S., Gallagher, P. T., et al. 2013, Sol. Phys., 286, 111

Rottman, G. J., Woods, T. N., \& McClintock, W. 2006, Adv. Space Res., 37, 201

Schmutz, W., Fehlmann, A., Finsterle, W., Kopp, G., \& Thuillier, G. 2013, AIP Conf. Ser., 1531, 624
Schrijver, C. J. 1983, A\&A, 127, 289

Schrijver, C. J., \& Harvey, K. L. 1989, ApJ, 343, 481

Seaton, D. B., Berghmans, D., Nicula, B., et al. 2013, Sol. Phys., 286, 43

Skumanich, A., Smythe, C., \& Frazier, E. N. 1975, ApJ, 200, 747

Solanki, S. K. 2003, A\&ARv, 11, 153

Stepanov, V. E., \& Petrova, N. N. 1959, Izv. Krymsk. Astrofiz. Observ., 21, 352

Tamhane, A., \& Dunlop, D. 2000, Statistics and Data Analysis: From Elementary to Intermediate (Pearson)

Verbeeck, C., Delouille, V., Mampaey, B., \& De Visscher, R. 2014, A\&A, 561, A29

Veselovsky, I. S., Zhukov, A. N., Dmitriev, A. V., et al. 2001, Sol. Phys., 201, 27 Wenzler, T., Solanki, S. K., Krivova, N. A., \& Fröhlich, C. 2006, A\&A, 460, 583

Worden, J. R., White, O. R., \& Woods, T. N. 1998, ApJ, 496, 998

Yalim, M. S., \& Poedts, S. 2014, Adv.n Astron., 2014, 957461

Yeo, K. L., Krivova, N. A., \& Solanki, S. K. 2014a, Space Sci. Rev., 186, 137

Yeo, K. L., Krivova, N. A., Solanki, S. K., \& Glassmeier, K. H. 2014b, A\&A, 570, A85 